\title{
Multilinear Algebras and Tensors with Vector Subbundle of Manifolds
}

\author{
Khondokar M. Ahmed ${ }^{1 *}$ and Saraban Tahora ${ }^{2}$ \\ ${ }^{1}$ Department of Mathematics, Dhaka University, Dhaka-1000, Bangladesh. \\ ${ }^{2}$ Department of Natural Sciences, University of Information Technology and Sciences, Dhaka-1212, Bangladesh.
}

(Received: 9 January 2013; Accepted: 23 September 2013)

\begin{abstract}
In the present paper some aspects of tensor algebra, tensor product, exterior algebra, symmetric algebra, module of section, graded algebra, vector subbundle are studied. A Theorem 1.32. is established by using sections and fibrewise orthogonal sections of an application of Gran-Schmidt.
\end{abstract}

Keywords: Multilinear and tensor algebra, tangent and tensor bundle, subbundle associated frame bundles, graded and Symmetric algebra.

\section{Introduction}

Multilinear algebra and tensor algebra of $R$-modules are needed to use higher order tensors. The tangent bundle, various tensor bundle, subbundle and associated frame bundles will play important roles as the theory of manifolds is developed. A theorem related with subbundle is treated with various tensor, graded algebra, tensor product, and trivial bundles.

\section{Tensor Algebra}

In order to study $R$-multilinear maps, we build a universal model of multilinear objects called the tensor algebra over $R$, where $R$ will be the ring $C^{\infty}(M)$.

Definition 1.1. An $R$-module $V$ is free if there is a subset $B \subset V$ such that every nonzero element $v \in V$ can be written uniquely as a finite $R$-linear combination of elements of $B$. The set $B$ will be called a (free) basis of $R$.

Example 1.2. Let $\pi: E \rightarrow M$ be a trivial $n$ - plane bundle [1]. Then $\Gamma(E)$ is a free $C^{\infty}(M)$

-module on a basis of $n$ elements. Another example is the integer lattice $\mathbb{Z}^{k}$, a free $\mathbb{Z}$-module.

Definition 1.3. If $V_{1}, V_{2}, V_{3}$ are objects in $\mathcal{M}(R)$,

a map $\varphi: V_{1} \times V_{2} \rightarrow V_{3}$ is $R$ - linear if

$\varphi\left(., V_{2}\right): V_{1} \rightarrow V_{3}$

$\varphi\left(V_{1},.\right): V_{2} \rightarrow V_{3}$

are $R$ - linear, $\forall v_{i} \in V_{i}, i=1,2$.

Definition 1.4. [2] A tensor product of $R-$ modules $V_{1}, V_{2}$ is an $R$-module $V_{1} \otimes V_{2}$, together with an $R$-bilinear map

$\otimes: V_{1} \times V_{2} \rightarrow V_{1} \otimes V_{2}$

with the following "universal property":

given any $R$-modules $V_{3}$ and any $R$-bilinear map

$\varphi: V_{1} \times V_{2} \rightarrow V_{3}$, there is a unique $R$-linear map $\tilde{\varphi}: V_{1} \otimes V_{2} \rightarrow V_{3}$ such that the diagram

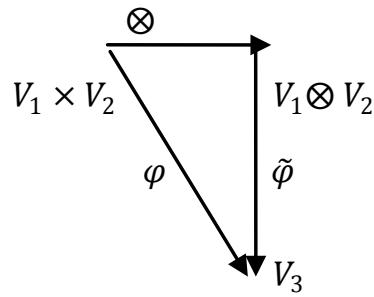

commutes. Write $\otimes(v, w)=v \otimes w$.

Corollary 1.5. If $V_{i}$ is an $R$-module, $i=1,2,3$, there are unique $R$-linear isomorphism

$$
\begin{aligned}
V_{1} \otimes\left(V_{2} \otimes V_{3}\right) & =\left(V_{1} \otimes V_{2}\right) \otimes V_{3} \\
& =V_{1} \otimes V_{2} \otimes V_{3}
\end{aligned}
$$

identifying

$$
\begin{aligned}
v_{1} \otimes\left(v_{2} \otimes v_{3}\right)= & \left(v_{1} \otimes v_{2}\right) \otimes v_{3} \\
= & v_{1} \otimes v_{2} \otimes v_{3}, \\
& \forall v_{i} \in V_{i}, \quad i=1,2,3 .
\end{aligned}
$$

Definition 1.6. An element $v \in V_{1} \otimes \ldots \otimes V_{k}$ is said to be decomposable if it can be written as a monomial $v=$ $v_{1} \otimes \ldots \otimes v_{k}$, for suitable elements $v_{i} \in V_{i}, 1 \leq i \leq$ $k$. Otherwise, $v$ is said to be indecomposable.

Lemma 1.7. If $V$ and $W$ are $R$-modules with respective bases $A$ and $B$, then $V \otimes W$ is free with basis $C=$ $\{a \otimes b \mid a \in A, b \in B\}$.

Proof. An arbitrary element $v \in A \otimes B$ can be written as a linear combination of decom-posable. A decomposable element $V \otimes W$ can be expanded the multilinearity of tensor product, to a linear combination of elements of $C$, proving that $C$ spans $V \otimes W$. It remains to show that, if

$$
\sum_{i, j=1}^{p, q} c_{i j} a_{i} \otimes b_{j}=\sum_{i, j=1}^{p, q} d_{i j} a_{i} \otimes b_{j},
$$


where $a_{i} \in A$ and $b_{j} \in B, 1 \leq i \leq p, 1 \leq j \leq q$ then all $c_{i, j}=d_{i, j}$. Subtracting one expression from the other, we only need to prove that

$\sum_{i, j=1}^{p, q} c_{i j} a_{i} \otimes b_{j}=0 \ldots$

implies that all $c_{i, j}=0$. The bilinear functional $\varphi: V \times$ $W \rightarrow R$ correspond one to one to any functions $f: A \times$ $B \rightarrow R$.The correspondence is $\varphi \leftrightarrow \varphi \mid(A \times B)$. Thus, the linear functionals $\tilde{\varphi}: V \otimes W \rightarrow R$ also correspond one to one to these functions $f: A \times B \rightarrow R$.

If $(a, b) \in(A \times B)$, let $f_{a, b}:(A \times B) \rightarrow R \quad$ be the function taking the value 1 on (a,b) and the value 0 on every other element of $(A \times B)$.

The corresponding linear functional will be denoted by $\tilde{\varphi}_{a, b}$. Applying $\tilde{\varphi}_{a_{i}, b_{j}}$ to equation (4.1), we see that all $c_{i j}=0$. This completes the proof.

Proposition 1.8. If $\lambda_{i}: V_{i} \rightarrow W_{i}$ is an $R-$

linear map, $1 \leq i \leq k$, there is a unique $R$-linear map

$$
\begin{aligned}
\lambda_{1} \otimes \ldots \ldots \otimes \lambda_{k}: V_{1} \otimes \ldots \ldots \otimes V_{k} \\
\\
\quad \rightarrow W_{1} \otimes \ldots \ldots \otimes W_{k}
\end{aligned}
$$

which, on decomposable elements, has the formula

$$
\begin{gathered}
\left(\lambda_{1} \otimes \ldots \ldots \otimes \lambda_{k}\right)\left(v_{1} \otimes \ldots \ldots \otimes v_{k}\right) \\
=\lambda_{1}\left(v_{1}\right) \otimes \ldots \ldots \otimes \lambda_{k}\left(v_{k}\right) .
\end{gathered}
$$

Proof. We know the decomposable span. So, the uniqueness is immediate. For existence, let us define the multilinear map

$\lambda: V_{1} \times \ldots \ldots \times V_{k} \rightarrow W_{1} \otimes \ldots \ldots \otimes W_{k}$

by

$\lambda\left(v_{1}, \ldots \ldots, v_{k}\right)=\lambda_{1}\left(v_{1}\right) \otimes \ldots \ldots . \lambda_{k}\left(v_{k}\right)$.

Then $\lambda_{1} \otimes \ldots \ldots . \otimes \lambda_{k}$ is defined to be the unique associated linear map. Hence, the proof is complete.

Definition 1.9. For the module of $R$-linear functionals, the dual $V^{*}$ of an $R$-module $V$ is $\operatorname{Hom}_{R}(V, R)$.

Lemma 1.10. If $V$ has a finite free basis $\left\{v_{1}, \ldots\right.$ $\left.\ldots \ldots, v_{n}\right\}$, then $V^{*}$ has a finite free basis $\left\{v_{1}, \ldots\right.$

$\left.\ldots \ldots, v_{n}\right\}$, called the basis and defined by $v_{i}^{*}\left(v_{j}\right)$

$=\delta_{j}^{i}, \quad 1 \leq i, j \leq n$.

Corollary 1.11. If $V_{1}, \ldots \ldots, V_{k}$ are free $R-$

modules on bases $B_{1}, \ldots \ldots, B_{k}$, respectively, then $V_{1} \otimes \ldots \ldots \otimes V_{k}$ is a free $R-$ module with basis

$B=\left\{v_{1} \otimes \ldots \ldots \otimes v_{k} \mid v_{i} \in B_{i}, \quad 1 \leq i \leq k\right\}$.

Proposition 1.12. There is a unique $R$-linear map

$l: V_{1}^{*} \otimes \ldots \ldots \ldots \otimes V_{k}^{*} \rightarrow\left(V_{1} \otimes \ldots \ldots \otimes V_{k}\right)^{*}$

which on decomposable elements has the formula

$$
\begin{aligned}
l\left(\eta_{1} \otimes \ldots \ldots \otimes \eta_{k}\right) & \left(v_{1} \otimes \ldots \ldots \otimes v_{k}\right) \\
= & \eta_{1}\left(v_{1}\right) \otimes \ldots \ldots \otimes \eta_{k}\left(v_{k}\right) .
\end{aligned}
$$

If the $R$-modules $V_{i}$ are all free on finite bases, then $l$ is a canonical isomorphism.

Proof. Since the decomposable span, uniqueness is immediate. For existence, consider the multi linear functional

$\theta: V_{1}^{*} \times \ldots \ldots \ldots \times V_{k}^{*} \times V_{1} \times \ldots \ldots \times V_{k} \rightarrow R$

by

$$
\begin{aligned}
\theta\left(\eta_{1}, \ldots \ldots, \eta_{k}, v_{1} \ldots \ldots, v_{k}\right) \\
\quad=\eta_{1}\left(v_{1}\right) \ldots \ldots \eta_{k}\left(v_{k}\right) .
\end{aligned}
$$

by the universal property, this gives the associated linear functional

$$
\tilde{\theta}: V_{1}^{*} \otimes \ldots \ldots \ldots \otimes V_{k}^{*} \otimes V_{1} \otimes \ldots \ldots \otimes V_{k} \rightarrow R,
$$

and we define

$l: V_{1}^{*} \otimes \ldots \ldots \ldots \otimes V_{k}^{*} \rightarrow\left(V_{1} \otimes \ldots \ldots \otimes V_{k}\right)^{*}$

by

$l(\eta)(v)=\tilde{\theta}(\eta \times v)$.

If $\left\{v_{i, 1}, \ldots \ldots, v_{i, m_{i}}\right\}$ is a basis of $V_{i}, 1 \leq i \leq k$, let

$\left\{v_{i, 1}^{*}, \ldots \ldots, v_{i, m_{i}}^{*}\right\}$ be the dual basis. Let $B$ and $B^{*}$

be the respective bases of $V_{1} \otimes \ldots \ldots \otimes V_{k}$ and $V_{1}^{*} \otimes \ldots \ldots \ldots \otimes V_{k}^{*}$ given by the Corollary 1.11. The formula

$$
\begin{array}{r}
l\left(v_{1, j_{1}}^{*} \otimes \ldots \ldots \otimes v_{k, j k}^{*}\right)\left(v_{1, i_{1}} \otimes \ldots \ldots \otimes v_{k, i_{k}}\right) \\
=\delta_{i_{1}}^{j_{1}} \ldots \ldots \delta_{i_{k}}^{j_{k}}=\delta_{i_{1} \ldots \ldots \ldots i_{k}}^{j_{1} \ldots \ldots j_{k}}
\end{array}
$$

shows that $l$ carries the basis $B^{*}$ one to one onto the basis dual to $B$, so $l$ is an isomorphism. This completes the proof.

Definition 1.13. [3] A graded (associated) algebra $A$ over $R$ is a sequence $\left\{A^{n}\right\}_{n=0}^{\infty}$ of $R$-modules, together with $R$-bilinear maps (multiplication)

$A^{n} \times A^{m} \rightarrow A^{n+m}, \quad \forall n, m \geq 0$,

which is strongly associative in the sense that the compositions

$$
\begin{aligned}
& \left(A^{n} \times A^{m}\right) \times A^{r} \stackrel{. \times i d}{\rightarrow} A^{n+m} \times A^{r} \stackrel{\dot{\rightarrow}}{\rightarrow n+m+r}, \\
& A^{n} \times\left(A^{m} \times A^{r}\right) \stackrel{i d \times .}{\longrightarrow} A^{n} \times A^{m+r} \stackrel{\dot{\rightarrow}}{\rightarrow} A^{n+m+r}
\end{aligned}
$$

are equal, $\forall n, m, r \geq 0$.

Definition 1.14. If $V$ is an $R$-module, then $\mathcal{T}(V)$ with multiplication $\otimes$, is called the tensor algebra of $V$. It is clear that the tensor algebra $\mathcal{T}(V)$ is connected.

Theorem 1.15. If $\lambda: V \rightarrow W$ is an $R$-linear map, then there is a unique induced homomorphism $\mathcal{T}(\lambda): \mathcal{T}(V) \rightarrow$ $\mathcal{T}(W)$ of graded $R$-algebras such that $\mathcal{T}^{0}(\lambda)=i d_{R}$ and $\mathcal{T}^{1}(\lambda)=\lambda$.

This homomorphism satisfies

$\mathcal{T}^{n}(\lambda)\left(v_{1} \otimes v_{2} \otimes \ldots \ldots \otimes v_{n}\right)$

$$
=\lambda\left(v_{1}\right) \otimes \lambda\left(v_{2}\right) \otimes \ldots \ldots . \otimes \lambda\left(v_{n}\right),
$$


$\forall n \geq 2, \forall v_{i} \in V, 1 \leq i \leq n$.

Finally, this induced homomorphism makes $\mathcal{T}$ a covariant function from the category of $R$-modules $R$-linear maps to the category of graded algebras over $R$ and graded algebra homomorphisms.

Definition 1.16. The space of tensors on $V$ of type $(r, s)$ is the tensor product

$\mathcal{T}_{s}^{r}(V)=\mathcal{T}_{0}^{r}(V) \otimes \mathcal{T}_{S}^{0}(V)$.

\section{Exterior Algebra}

The $R$-module $\Lambda^{k}(V)$ is called the $k$ th exterior power of $V$. The connected graded $R$-algebra

$$
\Lambda(\mathrm{V})=\left\{\Lambda^{k}(V)\right\}_{\mathrm{k}=0}^{\infty}
$$

with multiplication

$\Lambda^{p}(V) \times \Lambda^{q}(V) \stackrel{\Lambda}{\rightarrow} \Lambda^{p+q}(V)$

is called the exterior algebra of $V$ [4].

Lemma 1.17. Let $V$ be an $R$-module, $v \in V$. Then $v=-v \Leftrightarrow v=0$.

Proof. Let $V$ be an $R-$ module where $v \in V$. Then $v=0 \Rightarrow v=-v$.

For the converse

$$
\begin{gathered}
v=-v \Rightarrow 2 v=0 \\
\Rightarrow v=1 / 2(2 v) \\
\Rightarrow v=1 / 2(0) \\
\therefore v=0 .
\end{gathered}
$$

This completes the proof.

Definition 1.18. Let $V$ and $W$ be $R$-modules.

An antisymmetric $K$-linear map $\varphi: V^{k} \rightarrow W$ is a $K$ - linear map such that

$$
\begin{aligned}
& \varphi\left(v_{\sigma(1)}, \ldots \ldots \ldots, v_{\sigma(k)}\right) \\
& \quad=(-1)^{\sigma} \varphi\left(v_{1}, v_{2}, \ldots \ldots, v_{k}\right), \quad \forall v_{1}, v_{2}, \ldots \ldots, v_{k}
\end{aligned}
$$

$\in V, \forall \sigma \in \sum k$

where $(-1)^{\sigma}= \begin{cases}1, & \sigma \text { an even permutation, } \\ -1, & \sigma \text { an odd permutation. }\end{cases}$

Lemma 1.19. If $\varphi: V^{k} \rightarrow W$ is antisymmetric, then $\widetilde{\varphi}\left(\mathfrak{A}^{k}(V)\right)=\{0\}$.

Proof. It will be enough to show that $\widetilde{\varphi}$ vanishes on a set spanning $\mathfrak{A}^{k}(V)$. Thus, if $w \in \mathcal{T}^{p}(V)$,

$u \in \mathcal{T}^{q}(V), p+q=k-2$, and $v_{1}, v_{2} \in V$, we

will show that

$\widetilde{\varphi}\left(w \otimes\left(v_{1} \otimes v_{2}+v_{2} \otimes v_{1}\right) \otimes u\right)=0$.

But the antisymmetry of $\varphi$ implies that

$\widetilde{\varphi}\left(w \otimes v_{1} \otimes v_{2} \otimes u\right)=-\widetilde{\varphi}\left(w \otimes v_{2} \otimes v_{1} \otimes u\right)$, and the assertion follows the linearity.

Definition 1.20. An element $w \in \Lambda^{k}(V)$ that can be expressed in the form $v_{1} \wedge v_{2} \wedge \ldots \ldots \wedge v_{k}$, where $v_{i} \in V$, $1 \leq i \leq k$, is said to be decomposable. Otherwise, $w$ is indecomposable.

Definition 1.21. A graded algebra $A$ is anti commutative if $\alpha \in A^{k}$ and $\beta \in A^{r} \Rightarrow \alpha \beta=$ $(-1)^{k r} \beta \alpha$.

Corollary 1.22. [3] The graded algebra $\Lambda(\mathrm{V})$ is anticommutative.

Proof. It is enough to verify the Definition 1.20. for decomposable elements of $\Lambda^{k}(V)$ and $\Lambda^{r}(V)$. But that case is an elementary consequence of the case $k=r=1$, and this latter case is given by

$$
\begin{aligned}
v \wedge w & =v \otimes w+\mathfrak{A}^{2}(V) \\
& =w \otimes v+\mathfrak{I}^{2}(V) \\
& =-w \wedge v,
\end{aligned}
$$

$\forall v, w \in V$. Thus the graded algebra $\Lambda(\mathrm{V})$ is ticommutative. Corollary 1.23. If $w \in \Lambda^{2 r+1}(V)$, then $w \wedge w=0$.

Proof. Let $w \in \Lambda^{2 r+1}(V)$. Then

$$
\begin{aligned}
w \wedge w & =(-1)^{(2 r+1)(2 r+1)}(w \wedge w) \\
& =w \wedge w
\end{aligned}
$$

Now, by using Lemma 1.17., we have

$w \wedge w=0$.

This completes the proof

Lemma 1.24. If $\lambda: V \rightarrow V$ is linear, then $\Lambda^{m}(\lambda)$ : $\Lambda^{m}(V) \rightarrow \Lambda^{m}(V)$ is multiplication by $\operatorname{det}(\lambda)$.

Proof. Relative to a basis $\left\{e_{1}, \ldots \ldots, e_{m}\right\}$ of $V$, write

$\lambda\left(e_{i}\right)=\sum_{j=1}^{m} a_{i}^{j} e_{j}, \quad 1 \leq i \leq m$

then,

$$
\begin{aligned}
& \Lambda^{m}(\lambda)\left(e_{1} \wedge \ldots \ldots \wedge e_{m}\right) \\
& \quad=\lambda\left(e_{1}\right) \wedge \ldots \ldots \wedge \lambda\left(e_{m}\right) \\
& =\left(\sum_{j=1}^{m} a_{1}^{j} e_{j}\right) \wedge \ldots \ldots\left(\sum_{j=1}^{m} a_{m}^{j} e_{j}\right) \\
& =\sum_{1 \leq j_{1}, \ldots \ldots, j_{m \leq m}} a_{1}^{j_{1}} \ldots \ldots a_{m}^{j_{m}} e_{j_{1}} \wedge \ldots \ldots \wedge e_{j_{m}} .
\end{aligned}
$$

Any term with a repeated $j$ index vanishes. If $J=$ $\left(j_{1}, j_{2}, \ldots \ldots, j_{m}\right)$ contains no repetitions, there is a unique permutation $\sigma j \in \sum m$ such that

$j_{\sigma j}(r)=r, \quad 1 \leq r \leq m$. 
Thus,

$\Lambda^{m}(\lambda)\left(e_{1} \wedge \ldots \ldots \wedge e_{m}\right)$

$=\left(\sum_{\sigma \in \sum m}(-1)^{\sigma} a_{\sigma_{(1)}}^{1} \ldots \ldots a_{\sigma(m)}^{m}\right) e_{1} \wedge \ldots \ldots \wedge e_{m}$

$=\operatorname{det}(\lambda)\left(e_{1} \wedge \ldots \ldots \wedge e_{m}\right)$.

Hence, the proof is complete.

Lemma 1.25. If $R$ is a field, a set of vectors $w_{1}, w_{2}, \ldots \ldots, w_{k} \in V, k \geq 2$, is linearly indepen-

dent if and only if $w_{1} \wedge w_{2} \wedge \ldots \ldots \wedge w_{k} \neq 0$.

Proof. If $R$ is a field then consider the set of vectors $w_{1}, w_{2}, \ldots \ldots, w_{k} \in V, k \geq 2$. Again if the set is dependent, the existence of universe in $R$ allows us to assume, without loss of generality, that

$$
w_{1}=\sum_{i=2}^{k} a_{i} w_{i} \text {. }
$$

Then

$$
\begin{aligned}
& w_{1} \wedge w_{2} \wedge \ldots \ldots \wedge w_{k} \\
& \quad=\sum_{i=2}^{k} a_{i} w_{i} \wedge w_{2} \wedge \ldots \ldots \wedge w_{k}=0 .
\end{aligned}
$$

Conversely, if the set is linearly independent, extend it to a basis by suitable choices of $w_{k+1}, \ldots \ldots, w_{m} \in V$. Then, we have

$w_{1} \wedge w_{2} \wedge \ldots \ldots \wedge w_{k} \wedge \ldots \ldots \wedge w_{m}$

is a basis of the one-dimensional space $\Lambda^{m}(V)$, hence is not 0 .

This completes the proof.

Lemma 1.26. If $V$ is a free $R$-module on a finite basis, then each $A^{k}$ is one to one, hence $A: \Lambda(\mathrm{V}) \hookrightarrow \mathcal{T}(V)$ is a canonical graded linear imbedding.

Proof. Let $\left\{e_{1}, \ldots \ldots, e_{m}\right\} \subset V$ be a basis and consider the basis

$\left\{e_{i_{1}} \wedge \ldots \ldots \wedge e_{i_{k}}\right\}_{1 \leq i_{1} \leq \cdots \ldots<i_{k} \leq i_{m}}$

of $\Lambda^{k}(V)$. Let $\left\{e_{1}^{*}, \ldots \ldots, e_{k}^{*}\right\} \subset V^{*}$ be the dual basis. Since $\mathcal{T}^{k}\left(V^{*}\right)=\mathcal{T}^{k}(V)^{*}$, we obtain a subset

$\left\{e_{j_{1}}^{*} \otimes \ldots \ldots \otimes e_{j_{k}}^{*}\right\}_{1 \leq j_{1}<\cdots \ldots<j_{k} \leq j_{m}} \subset \mathcal{T}^{k}(V)^{*}$,

which is a part of a free basis. Then, since $j_{1}<\cdots<j_{k}$ and $i_{1}<\cdots<i_{k}$,

$\left(e_{j_{1}}^{*} \otimes \ldots \ldots \otimes e_{j_{k}}^{*}\right)\left(A^{k}\left(e_{i_{1}} \wedge \ldots \ldots \wedge e_{i_{k}}\right)\right)$

$$
\begin{aligned}
& =\left(e_{j_{1}}^{*} \otimes \ldots \ldots \otimes e_{j_{k}}^{*}\right)\left(\sum_{\sigma \in \sum k}(-1)^{\sigma} e_{i_{\sigma(1)}} \otimes \ldots \ldots \otimes e_{i_{\sigma(k)}}\right) \\
= & \left(e_{j_{1}}^{*} \otimes \ldots \ldots \otimes e_{j_{k}}^{*}\right)\left(e_{i_{1}} \otimes \ldots \ldots \otimes e_{i_{k}}\right) \\
= & \delta_{i_{1} \ldots i_{k}}^{j_{1} \ldots j_{k}}
\end{aligned}
$$

and the assertion follows.

\section{Symmetric Algebra}

A $K$-linear map $\varphi: V^{k} \rightarrow W$ is symmetric if, for each $\sigma \in \sum k$,

$\varphi\left(v_{\sigma(1)}, \ldots \ldots, v_{\sigma(k)}\right)=\varphi\left(v_{1}, v_{2}, \ldots \ldots, v_{k}\right)$,

$$
\forall v_{1}, v_{2}, \ldots \ldots, v_{k} \in V .
$$

In the usual way, we build a universal, symmetric, $K$-linear map

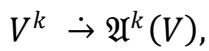

Usually written with the dots $\left(v_{1}, v_{2}, \ldots \ldots, v_{k}\right) \mapsto v_{1} v_{2} \ldots \ldots v_{k}$.

Definition 1.27. [5] The space $\mathfrak{A}^{k}(V)$ is called the $k$ th symmetric power of $V$, where, as usual, $\mathfrak{\mathfrak { I }}^{0}(V)=$ $R$ and $\mathfrak{A}^{1}(V)=V$. The connected, graded algebra $\mathfrak{A}(V)=$ $\left\{\mathfrak{A}^{k}(V)\right\}_{k=0}^{\infty}$, with multiplication ". ", is called the symmetric algebra of $V$.

Definition 1.28. Let $V$ be a finite dimensional vector space over a field $\mathbb{F}$. A function $f: V \rightarrow \mathbb{F}$ is a homogeneous polynomial of degree $k$ on $V$ if, related to some basis $\left\{e_{1}, \ldots \ldots, e_{m}\right\}$ of $V$,

$f\left(\sum_{i=1}^{m} x_{i} e_{i}\right)=P\left(x_{1}, \ldots \ldots, x_{m}\right)$

is a homogeneous polynomial of degree $k$ in the variables $x_{1}, \ldots \ldots, x_{m}$. The vector space of all homogeneous polynomials of degree $k$ on $V$ will be denoted by $P^{k}(V)$.

\section{The Module of Sections}

We are going to view the set of all vector bundles over a fixed manifold $M$ [5] as the objects of a category $V_{M}$. Let

$\pi: E \rightarrow M$

$\rho: F \rightarrow M$

be vector bundles (of possibly differing fibers dimensions). A homomorphism of the $n$-plane bundle $E$ to the $m$-plane bundle $F$ is denoted by $\operatorname{HOM}(E, F)$ is naturally called $C^{\infty}(M)$ - module.

Theorem 1.29. [5] The $C^{\infty}(M)$-linear map $\alpha$ is a canonical isomorphism of $C^{\infty}(M)$ - modules.

$$
\Gamma(E) \otimes_{C^{\infty}(M)} \Gamma(F)=\Gamma(E \otimes F) .
$$


Corollary 1.30. [5] There are canonical iso- morphisms $C^{\infty}(M)$ - modules

$\Gamma\left(\mathcal{T}^{k}(E)\right)=\mathcal{T}^{k}(\Gamma(E))$

$\Gamma\left(\Lambda^{k}(E)\right)=\Lambda^{k}(\Gamma(E))$

$\Gamma\left(S^{k}(E)\right)=S^{k}(\Gamma(E))$.

Proof. The first part of these identities is an immediate consequence of theorem 1.29. There is canonical inclusion

$A^{k}: \Lambda^{k}(\Gamma(E)) \hookrightarrow \mathcal{T}^{k}(\Gamma(E))$

$A^{k}: \Gamma\left(\Lambda^{k}(E)\right) \hookrightarrow \Gamma\left(\mathcal{T}^{k}(E)\right)$.

The second part comes from the bundle inclusions. The images of these inclusions correspond perfectly under the identification $\mathcal{T}^{k}(\Gamma(E))=\Gamma\left(\mathcal{T}^{k}(E)\right)$, proving the second identity. Similarly the third part can be proof which is same as proof of second part.

Lemma 1.31. If $\mathrm{F}$ and $E$ are trivial bundles, then $\alpha$ is an isomorphism of $C^{\infty}(M)$ - modules.

Proof. In this case we choose the global sections $\left\{\sigma_{1}, \ldots \ldots, \sigma_{n}\right\}$ of $E$ and $\left\{\mathcal{T}_{1}, \ldots \ldots, \mathcal{T}_{m}\right\}$ of $F$ which trivialize these bundles. These are free bases of the respective $C^{\infty}(M)-$ modules $\Gamma(E)$ and $\Gamma(F)$, so

$\left\{\sigma_{i} \otimes_{C^{\infty}(M)} \mathcal{T}_{j}\right\}_{i, j=1}^{n, m}$

is a free basis of $\Gamma(E) \otimes_{C^{\infty}(M)} \Gamma(F)$. The set

$\left\{\sigma_{i} \otimes \mathcal{T}_{j}\right\}_{i, j=1}^{n, m}$

of point wise tensor products of sections trivializes the bundle $E \otimes F$, hence this is also a free basis of $\Gamma(E \otimes F)$. Since

$\alpha\left(\sigma_{i} \otimes_{C^{\infty}(M)} \mathcal{T}_{j}\right)=\sigma_{i} \otimes \mathcal{T}_{j}$,

for all relevant indices, we see that $\alpha$ is an isomorphism of $C^{\infty}(M)$ - modules. This completes the proof.

Theorem 1.32. If $F \subseteq E$ is a vector subbundle and if there is given Riemannian metric on $E$, then the subset $\tilde{F} \subseteq E$, fiber wise perpendicular to $F$, is a subbundle.
Proof. Here the local triviality all that needs to be proven. There are sections $\sigma_{1}, \ldots \ldots, \sigma_{r}, \sigma_{r+1}$,

$\ldots \ldots, \sigma_{n}$ of $E \mid U$, trivializing that bundle, where $U$ is a neighborhood of an arbitrary point of $M$. These can be chosen so that $\sigma_{1}, \ldots \ldots, \sigma_{r}$ are sections of $F \mid U$ which trivialize that bundle an application of Gran-Schmidt turns these into fiberwise orthonormal sections $S_{1}, \ldots \ldots, S_{r}, S_{r+1}$

$, \ldots \ldots, S_{n}$ with the same properties. It follows that $S_{r+1}, \ldots \ldots, S_{n}$ are trivializing sections of $\tilde{F} \mid U$, proving that $\tilde{F}$ is a subbundle of $E$. Hence the proof is complete.

\section{Conclusion}

A theorem 1.32 is established which is related with a Riemannian metric on the bundle $M \times V$. For each $x \in M$, let $\tilde{E}_{x} \subset\{x\} \times V$ be the subspace orthogonal to $E_{x}^{\perp}$. Consequently the set $\tilde{E}=\bigcup_{x \in M} \tilde{E}_{x}$ is a subbundle of $M \times V$. Also this theorem will follow form a theorem in dimension theory.

\section{References}

1. Boothby, W., 1975. An Introduction to Differentiable Manifolds and Differential Geometry, Academic Press, NewYork..

2. Donson, C. T. J. and T. Poston, 1997.Tensor Geometry, Pitman, London.

3. Ahmed, K. M., 2007. A study of Graded manifolds, Dhaka Uni. J. Sci. 55 (1): 35-39.

4. Chevally, C. 1956. Fundamental Concepts of Algebra, Academic Press, New York.

5. Myers, S. B. and N. E., 1939.Steenrod, the group of isometrics of a Riemannian Manifold, Ann of Math. 40: 400-416.

6. Auslander, L. and R. E. Mackenzie, 1963. Introduction to differential Manifolds, Mc Graw-Hill, New York.

7. Brickell, F. and R. S. Clark, 1970. Differential Manifolds, Van Nostrand Reinhold company, London. 